4. The face and eyes appear swelled, which was supposed to be caused by the child sleeping near the door. The bowels being constipated, a powder, containing two grains of calomel and five of jalap, was ordered.

5. The lower part of the face more enlarged; the integument has a shining and glazed appearance; the child sleeps much; bowels opened twice from the powder; the body is much emaciated. Saline medicine to be taken occasionally.

6. Has been extremely restless during the night; rolling the hear continually; the right cheek very hard; the mouth can be opened, and the tongue protruded, which is furred; the pulse cannot be counted, in consequence of the uneasiness of the arms, which are constantly tossed about, and placed to the face, as if suffering pain; a blister to be applied to the posterior part of the neck.

7. There is a large ulcer on the inner surface of the right cheek, from which there is a most unpleasant odour, as if from a mortified part; the bowels are relaxed; a gargle of decoction of bark and albumen to be syringed into the mouth frequently; three grains of quinine and two of chalk to be given every four hours.

8. The gangrene is much increased, extending to the gums; the left side of the mouth more inflamed; tongue perfectly natural; the teeth on the right side of the lower jaw loose; the mother, on applying the syringe, pressed out the two lower incisors; debility appears coming on fast; a grain of quinine to be given in port-wine every hour.

10. The gangrene on the right side much more extensive; there is a blue mark, of the size of a shilling, on the exterior of the cheek, as if the sphacelus had almost passed throngh the integuments; the child was delirious in the night, and hit her face with her hands most violently; the evacuations are passed involuntary; an ounce of honey to be mixed with 60 drops of muriatic acid, and applied to the gangrenous surface on the inner parts of the mouth. Death took place during the night; the friends would not permit the body being examined; but on introducing the finger into the mouth, nearly the whole of the right side appeared destroyed as far as the finger could reach; the left was little affected.

\section{IDIOPATHIC GANGRENE} OF THE

MOUTH; DISEASE OF THE MOUTH FROM

UNFIT OR DEFICIENT FOOD.

To the Editor of THE LANCET.

SIR:-As gangrene of the mouth assumes, at the present time, a degree of consequence and interest, superadded to that which its original horribleness has obtained for it, $I$ transmit to you the narrative of two cases of a different description of that disease from those related by your contributors in last week's LANCET.

Sept. 6, 182S. Mary Parkinson, æt. six years, resides at Paddock; had hooping. cough for more than three weeks, but she is now quite cured; about a week since became rather feverish and thirsty, and lost her appetite; bowels confined; complains of pain in the bowels and headach; pulse 100 ; tongue moist, rather white; was a remarkably healthy child until the attack of hooping-cough. Prescribed-compound jalap powder, a scruple night and morning, and a saline mixture.

10. Contiuued nearly the same until to. day, every symptom being nearly as before; the right side of the mouth and cheek are rather swollen, and have a slight blush of a dark red colour; she endeavours to scrateh and pull at these inflamed parts; no ulcera. tion, or other disease, or inflammation of the membrane of the mouth or fauces; pulse 110 ; tongue moist, and corered with a white fur; skin nearly the natural warmth, moist, and soft ; urine passed freely; botvels freely opened. Prescribed-fomentation: bread poultice; saline mixture, with magnesia and manna, and milk diet.

12. Inflammation of the cheek, and mouth much extended, and the colour a darker red; a black spot, of the size of a pea, is apparent upon the external surface of the right cheek, near to the angle of the month; pulse 110, small, and rather jerking; lower gum rather iswollen, and a darker colour than natural, but in other respects the interior of the mouth differs not from its usual appearance; tongue dry, and covered with a thin brown fur; endeavours to scratch and pull her face and mouth. Hot bread and milk poultices repeated every two or three hours; cinchona mixture, with ammonia; beef-tea; a little beer or wine fre. quently.

13. Right cheek all over red; both hips red and swollen, principally the lower; black spot, the size of a shilling, extends to the angle of the mouth; bowels act freely; kidneys secrete a sufficient quantity of urine; she takes a little beer, and some bread and milk; has not been sick, nor delirious.

15. Slough nearly the size of half-a-crown piece, extends into the lower lip, and occupies the entire thickness of the cheek; it feels like a piece of leather; no discharge; chloride of soda wash, and yeast poultices; decoction of cinchona and ammonia, and whatever food and wine can be taken.

17. Gangrene extends nearly half over the lower lip, up the cheek as far as the nose and molar bone, and half over the cheek posteriorly; the lower gum on the 
right side a dark brown, and separating from the teeth; a thin fotid discharge oozes from the part; throat and tongue not affected, the latter harsh and dry, and covered with a dark-brown fur; very thirsty; drowsy, yet restless and fidgety; sometimes talks incoherently; tries to tear her face, and cries violently if withheld; pulse 125, small, and weak; complains of pain in the bowels; motions and urine passed invoIuntarily.

21. Gangrene occupies the whole of both lips, as far as below the chin and up to the nose, and about an inch beyond the left angle of the mouth, and nearly as far as the angle of the jaw on the right side; also the upper and lower gums, whence several teeth have either dropped or been picked out; the part exhales a very disagreeable stench; urine and motions passed involuntarily; diarrhœa; very offensive dark-coloured motions; sometimes vomits; low muttering delirium; continually picking and tearing her face; pulse 140, very small, and feeble; a large portion of the lower lip, which hung loosely over the chin, I clipped off, in order to diminish the offensive smell.

24. The patient died this morning. She had been very delirious for two days, and had a bad diarrhoea; pulse ranged from 140 to 160. Gangrene extends on the right side nearly to the arm, some distance under the chin, and up to the eye; on the left cheek, as far as the anterior edge of the masseter; occupies the upper lip and cartilege of the nose, all the gums from which the teeth have all come out, with the exception of a few at the back on the left side, and extends under the tongue. After this description, it will be hardly necessary to say that a more loathsome object can scarcely be imagined. I am sorry that no post-mortem could be obtained of this or the following case, as there was at that time in Huddersfield a very great and quite insurmountable prejudice against every thing of the kind.

May 14, 1829. Ann Green, æt. seven years, a tall, healthy-looking girl, rather thin; lives at Hill-house; has complained of headach for some weeks; but she did not appear to be ill until the 8th of May; since then her appetite has been impaired, and she has been thirsty, and rather feverish; sometimes pale, and at other times flushed in the face; has not been sick; headach not considerable; bowels not been opened for three days; complains of pain at the scrobiculus cordis, but it is not sore on pressure; perspires when asleep; restless nights; tongue covered with a thin white fur; pulse 95, with a slight thrill; an exacerbation of fever generally takes place in the evening; no wandering of the mind; was remarkably well up to the present illness; had measles and small-pox when about two years old, has not suffered from any illness since that time. Prescribed-senna and salts mix- ture, a dose every two hours until it ope. rates; saline mixture, with antimonial wine. Diet to be milk and water and tea.

15. Bowels freely opened; symptons nearly as before.

20. The patient seemed to be going on well until yesterday evening, when the left cheek was observed to be inflaned; exactly in the centre of the left cheek is a dark red spot, which she says itches and burns; there is no ulceration or inflamed appearance in the inside of the cheek, or any part of the mouth or throat; she complains of headach, and pain at the scrobiculus cordis, but the latter is not much increased by pressure; no vomiting; has two or three motions a day, which are dark-coloured and offensive; pulse 110; skin warmer than natural, but moist; tongue white, with a streak of brown in the centre; restless nights; often wakes up suddenly, and in a fright, thinking she sees persons about the bed; appetite very good; applied caustic all round the inflamed part. Prescribed - quinine, with dilute sulphuric acid; bread and water poultice; beer; beef tea.

21. Left cheek more swollen and in. flamed; there is a black speck appearing in the centre; symptoms nearly the same; she evidently does not feel very ill, as she walks about the house, and is tolerably cheerful. Made a large circle of caustic around the part ; continue quinine and poultices.

23. An eschar, of the size of half-a.crown, occupies the centre of the cheek, which feels like a piece of leather; inflammation has extended to the mouth and gums, which are swollen; was delirious in the night, but she is now quite sensible; bowels opened twice; tongue covered with brown fur; palse 120, small, and vibrating; headach; perspired freely in the night. Nitric acid solution; ung. terebinth. on the eschar, and yeast ponltices; decoct. cinchonæ, with am. monia; wine or beer, and any nutriment.

25. Gangrene extends to the nose, mouth, and lower edge of the jaw, and the gums on the left side; an offensive discharge; it does not affect the throat or tongue; con. tinue remedies.

26. Delirious all night, during which state she tore a large rent in her cheek, and pulled some teeth out; screams and cries continually; motions and urine passed invo. luntarily; gangrene ex tends rapidly; tongue dry and bown; pulse 140, very feeble.

27. Had convulsions in the night, in one of which she died. Post-mortem not per. mitted.

Remarks.-These two are the only in. stances of the idiopathic gangrene of the mouth which I have seen. Their causes, after the most careful inquiry, I am unable to assign with any degree of cerlainty. Both children were naturally healthy; their pa* rents, brothers, and sisters, were as fine healthy-looking persons as any in their 
neighbourhood: Parkinson lived in a populous part, in a cottage, in front of which flowed a deep rivulet, so that it may have been damp; but Green resided in an exactly opposite direction, in a beautifully rural situation, high and dry, and at some distance from other houses: locality, therefore, it may be supposed, had nothing to do with the occasion. The early symptoms were not unlike an attack of fever; but as typhoid or eruptive fevers were not then prevalent, it is not likely that it was a va. riety of that kind of fever. Death in both cases was produced by cerebral disease; but that state evidently supervened upon, and was occasioned by, an antecedent malady. The mucous membrane of the bowels was certainly diseased in the first case, and I think there must have been a morbid state of some of the chylo-poietic organs in the second case, from the complaints of pain about the scrobiculus cordis, and the unhealthy-looking motions. This disease is manifestly very different to that which succeeds small-pox, and similar malignant fevers, as in instances arising from these causes the malady is usually preceded by symptoms of a more or less aggravated form of ptyalism, and always commences in the gums and membrane of the cheek and mouth; it seems, indeed, to be a natural and inevitable result of the highly morbid state of the system, induced by these virulent diseases. Idiopathic gangrene of the mouth may be produced by improper diet in a child, imbued with a taint of scrofula, and perhaps under the influence of some disease lurking in the chest or abdomen.

There is an affection of the month in children, by no means uncommon, which, $\mathrm{I}$ cannot doubt, arises from an undue quantity, or a bad quality, of food. In this com. plaint the gums are red and spongy, and bleed when touched. In bad cases, the teeth fall out; the tongue swells, but not to a great degree; it is covered with a white fur, and large superficial ulcers are observed on the upper and under surfaces of the tongue, and the inside of the cheeks, which are marked by the teeth. The lips swell, and saliva flows from the mouth; pulse a little accelerated; skin not much warmer than natural; sometimes the perspirations are profuse. In some cases $\mathbf{I}$ have seen vomiting and diarrhoea; but sometimes the bowels are difficult to move. The patient frequently does not seem to be particularly ill, and, indeed, not uncommonly looks stout and ruddy, although the mouth and gums are very much ulcerated and diseased. You naturally ask, if mercury has been given; and the answer frequently is, that no medicine whatever has been administered. The next inquiry is about his diet, and you are informed that he eats and drinks whatever he likes best, which may sometimes happen to be substantial meals of meat and beer. I have always found two or three calomel and jalap powders, a saline mixture, and carbonate of potash, and a mild nitric acid wash, succeeded sometimes by a weak infusion of gentian, speedily re-establish the health, and remove the affection of the mouth.

It does not seem improbable that mercurials, when incautiously administered, may sometimes be a cause of gangrene of the mouth; and we may imagine that the most favourable condition for its development will exist in the last stages of malignant fevers, or when the constitution is enfeebled, or depraved, from any cause : it is, therefore, necessary to be extremely circumspect. As it has been clearly ascertained that the disease, in its worst form, occurs in children to whom not a single grain of any mercurial medicine has been administered, it would be very unfair in any case to assign that medicine as the cause, unless a considerable quantity had been given, or such a quantity as, considering all the circumstances, might plainly be sufficient to produce such a disastrous result.* I remain, Sir, your obedient servant,

WM. Baintridge, M.R.C.S.

Upper Tooting, Dec. 14, 1839.

\section{WESTERN GENERAL DISPENSARY,} NEW ROAD.

\section{To the Editor of THE LANCET.}

SIR:-A new mode of operating for tumours in the popliteal space has been discovered, which, for simplicity and dispatch, beats every thing hollow. Thrust an abscess lancet in; let it bleed freely until the patient looks droll, and you become frightened; then send off in a hurry for Sir Benjamin Brodie to witness your success, which he admires very much, by the elegant mode of putting his tongue in his cheek. Yours truly,

WASP.

* If the above cases of Mr. Bainbridge have been carefully observed, these are the only ones, out of several hundred cases of gaugrene of the mouth, in which the disease commenced on the cutaneous surface of the cheek.-ED. L. 